\title{
ON THE SOLUTION OF THE EQUATIONS OF ELASTIC EQUILIBRIUM SUITABLE FOR ELLIPTIC BOUNDARIES*
}

\author{
BY \\ SUDDHODAN GHOSH
}

1. The solution of the problem of the equilibrium of an isotropic elastic body with a given boundary depends, in the absence of body forces, on the solution of the partial differential equations

$$
\begin{aligned}
& (\lambda+\mu) \frac{\partial \Delta}{\partial x}+\mu \nabla^{2} u=0 \\
& (\lambda+\mu) \frac{\partial \Delta}{\partial y}+\mu \nabla^{2} v=0 \\
& (\lambda+\mu) \frac{\partial \Delta}{\partial z}+\mu \nabla^{2} w=0
\end{aligned}
$$

where

$$
\Delta=\frac{\partial u}{\partial x}+\frac{\partial v}{\partial y}+\frac{\partial w}{\partial z}
$$

subject to given conditions at the boundary. But the determination of the three functions $u, v, w$ satisfying equations (1) and (2) and the conditions at the bounding surface is a matter of great difficulty and hitherto such functions have been discovered only in the case of a few boundaries. It frequently happens, as with the kindred problems in hydrodynamics and electrostatics, that a problem which cannot be solved in three dimensions readily admits of a two-dimensional solution. Though these two-dimensional analogues can only be imperfectly produced in nature, they are of great importance on account of the light they throw on the more general problems which cannot be solved completely. From this point of view, the solutions of equations (1) and (2) in two dimensions are interesting and instructive and have actually received the attention of a large number of investigators.

It was shown by Airy that in the case of plane strain, the stresses can be expressed in terms of a stress function $\chi$ by the formulas.

$$
X_{x}=\frac{\partial^{2} \chi}{\partial y^{2}}, Y_{y}=\frac{\partial^{2} \chi}{\partial x^{2}}, X_{y}=-\frac{\partial^{2} \chi}{\partial x \partial y},
$$

* Presented to the Society, February 25, 1928; received by the editors in January, 1928. 
which in the absence of body forces further satisfies the partial differential equation of the fourth order

$$
\nabla_{1}^{4} \chi=0
$$

where

$$
\nabla_{1}^{4}=\nabla_{1}^{2} \cdot \nabla_{1}^{2} \text { and } \nabla_{1}^{2}=\frac{\partial^{2}}{\partial x^{2}}+\frac{\partial^{2}}{\partial y^{2}} .
$$

Consequently, equation (4) has received a considerable amount of attention from various mathematicians. The solutions in cartesian coördinates and their application to the problem of flexure of rectangular and cylindrical beams are well known. Those in polar coördinates have been given by Michell* and have been applied to plane bodies with concentric circular boundaries. Lately, a solution in bipolar coördinates has also been given $\uparrow$ thus opening the way of the consideration of areas bounded by two non-concentric circles. In the present paper a solution in elliptic coördinates has been found and applied to two problems of plane strain. A single-valued solution is found suitable for the case of an elliptic cylindrical cavity in an infinite solid while another many-valued solution applicable to the state of strain in an elliptic cylindrical shell which has suffered dislocation due to a triangular axial fissure has also been found. Love $\ddagger$ has given an outline of a method of solving problems in plane strain in elliptic coördinates, but his method is only applicable to cases where the surface displacements are given. The method developed in this paper lends itself easily to the construction of the solution of the particular problem dealt with by Love, as will be shown in $\S 5$. Some other applications of elliptic coördinates are given by Kolosoff, $\S$ Pöschl, \| and Inglis. $\Uparrow$

2. Let us use the transformation given by

$$
x+i y=c \cosh (\alpha+i \beta),
$$

so that

$$
x=c \cosh \alpha \cos \beta, \quad y=c \sinh \alpha \sin \beta,
$$

* Proceedings of the London Mathematical Society, vol. 31 (1900), p. 100.

$\dagger$ Jeffery, Philosophical Transactions, Royal Society, (A), vol. 221, pp. 265-293.

$\ddagger$ Theory of Elasticity (3d edition), p. 275.

\& Zeitschrift für Mathematik und Physik, vol. 62 (1914), p. 384.

|| Mathematische Zeitschrift, vol. 11 (1921), p. 89.

If Transactions of the Institute of Naval Architects, vol. 55, part 1, p. 219. My attention was drawn to Inglis's paper by one of the referees with the remark that it would be interesting to compare the expressions obtained in $\$ 4$ of this paper with those obtained by Inglis; but unfortunately as the paper was not available here no such comparison could be made. 
and

$$
\frac{1}{h^{2}}=\left(\frac{\partial x}{\partial \alpha}\right)^{2}+\left(\frac{\partial y}{\partial \alpha}\right)^{2}=\frac{c^{2}}{2}(\cosh 2 \alpha-\cos 2 \beta) .
$$

The curves $\alpha=$ constant and $\beta=$ constant are confocal ellipses and hyperbolas respectively. The curve $\alpha=0$ is the portion of the $x$-axis between the foci, while on the right-hand side of the $x$-axis between the focus and infinity, $\beta=0$, and on the left-hand side between the other focus and infinity, $\beta=\pi$. On the positive side of the $y$-axis $\beta=\pi / 2$ and on the negative side $\beta=3 \pi / 2$.

We have therefore a set of coördinates suitable for the treatment of twodimensional problems in which the region concerned is bounded by confocal ellipses and hyperbolas.

The stress components are given by equation (3). Transforming into curvilinear coördinates $(\alpha, \beta)$, the stress system can be written as

$$
\begin{aligned}
& \overparen{\alpha \alpha}=h \frac{\partial}{\partial \beta}\left(h \frac{\partial \chi}{\partial \beta}\right)-h \frac{\partial h}{\partial \alpha} \frac{\partial \chi}{\partial \alpha} \\
& \overparen{\beta \beta}=h \frac{\partial}{\partial \alpha}\left(h \frac{\partial \chi}{\partial \alpha}\right)-h \frac{\partial h}{\partial \beta} \frac{\partial \chi}{\partial \beta} \\
& \overparen{\alpha \beta}=-h \frac{\partial}{\partial \alpha}\left(h \frac{\partial \chi}{\partial \beta}\right)-h \frac{\partial h}{\partial \beta} \frac{\partial \chi}{\partial \alpha} .
\end{aligned}
$$

In the present case we have

$$
\begin{aligned}
\frac{\widehat{\alpha \alpha}}{h^{4}} & =\frac{1}{h^{2}} \frac{\partial^{2} \chi}{\partial \beta^{2}}+\frac{1}{2} \frac{\partial}{\partial \alpha}\left(\frac{1}{h^{2}}\right) \frac{\partial \chi}{\partial \alpha}-\frac{1}{2} \frac{\partial}{\partial \beta}\left(\frac{1}{h^{2}}\right) \frac{\partial \chi}{\partial \beta} \\
& =\frac{c^{2}}{2}\left[(\cosh 2 \alpha-\cos 2 \beta) \frac{\partial^{2} \chi}{\partial \beta^{2}}+\sinh 2 \alpha \frac{\partial \chi}{\partial \alpha}-\sin 2 \beta \frac{\partial \chi}{\partial \beta}\right] \\
\frac{\widehat{\beta \beta}}{h^{4}} & =\frac{1}{h^{2}} \frac{\partial^{2} \chi}{\partial \alpha^{2}}-\frac{1}{2} \frac{\partial}{\partial \alpha}\left(\frac{1}{h^{2}}\right) \frac{\partial \chi}{\partial \alpha}+\frac{1}{2} \frac{\partial}{\partial \beta}\left(\frac{1}{h^{2}}\right) \frac{\partial \chi}{\partial \beta} \\
& =\frac{c^{2}}{2}\left[(\cosh 2 \alpha-\cos 2 \beta) \frac{\partial^{2} \chi}{\partial \alpha^{2}}-\sinh 2 \alpha \frac{\partial \chi}{\partial \alpha}+\sin 2 \beta \frac{\partial \chi}{\partial \beta}\right] \\
\frac{\alpha \beta}{h^{4}} & =-\frac{1}{h^{2}} \frac{\partial^{2} \chi}{\partial \alpha \partial \beta}+\frac{1}{2} \frac{\partial}{\partial \alpha}\left(\frac{1}{h^{2}}\right) \frac{\partial \chi}{\partial \beta}+\frac{1}{2} \frac{\partial}{\partial \beta}\left(\frac{1}{h^{2}}\right) \frac{\partial \chi}{\partial \alpha} \\
& =\frac{c^{2}}{2}\left[-(\cosh 2 \alpha-\cos 2 \beta) \frac{\partial^{2} \chi}{\partial \alpha \partial \beta}+\sinh 2 \alpha \frac{\partial \chi}{\partial \beta}+\sin 2 \beta \frac{\partial \chi}{\partial \alpha}\right] .
\end{aligned}
$$

The displacements are given by*

* Jeffery, loc. cit. 


$$
2 \mu \frac{u}{h}=-\frac{\partial \chi}{\partial \alpha}+\frac{1}{h^{2}} \frac{\partial P}{\partial \beta}, \quad 2 \mu \frac{v}{h}=-\frac{\partial \chi}{\partial \beta}+\frac{1}{h^{2}} \frac{\partial P}{\partial \alpha},
$$

where $P$ satisfies the equations

$$
\nabla_{1}^{2} P=0
$$

and

$$
\frac{\partial}{\partial \alpha}\left(\frac{1}{h^{2}} \frac{\partial P}{\partial \beta}\right)+\frac{\partial}{\partial \beta}\left(\frac{1}{h^{2}} \frac{\partial P}{\partial \alpha}\right)=\frac{\lambda+2 \mu}{\lambda+\mu}\left(\frac{\partial^{2} \chi}{\partial \alpha^{2}}+\frac{\partial^{2} \chi}{\partial \beta^{2}}\right) .
$$

The stress function noted above satisfies equation (4).

3. Let us now proceed with the solution of equation (4). Writing it in the form

we notice that

$$
h^{2}\left(\frac{\partial^{2}}{\partial \alpha^{2}}+\frac{\partial^{2}}{\partial \beta^{2}}\right) h^{2}\left(\frac{\partial^{2} \chi}{\partial \alpha^{2}}+\frac{\partial^{2} \chi}{\partial \beta^{2}}\right)=0
$$

is a plane inarmonic function.

$$
h^{2}\left(\frac{\partial^{2} \chi}{\partial \alpha^{2}}+\frac{\partial^{2} \chi}{\partial \beta^{2}}\right)
$$

We now take the following form for the harmonic:

$h^{2}\left(\frac{\partial^{2} \chi}{\partial \alpha^{2}}+\frac{\partial^{2} \chi}{\partial \beta^{2}}\right)=\frac{2}{c^{2}}\left[A_{0}+A \alpha+B \beta+2 \sum_{n=1}^{n=\infty}\left(A_{n} \cosh n \alpha+B_{n} \sinh n \alpha\right) \cos n \beta\right.$

$$
\left.+2 \sum_{n=1}^{n=\infty}\left(C_{n} \cosh n \alpha+D_{n} \sinh n \alpha\right) \sin n \beta\right] \text {. }
$$

\section{Hence}

$\frac{\partial^{2} \chi}{\partial \alpha^{2}}+\frac{\partial^{2} \chi}{\partial \beta^{2}}=(A \alpha+B \beta)(\cosh 2 \alpha-\cos 2 \beta)+\left(A_{0}-A_{2}\right) \cosh 2 \alpha-B_{2} \sinh 2 \alpha$ $+\left\{-2 B_{1} \sinh \alpha+\left(A_{1}-A_{3}\right) \cosh 3 \alpha+\left(B_{1}-B_{3}\right) \sinh 3 \alpha\right\} \cos \beta$ $+\left\{2 C_{1} \cosh \alpha+\left(C_{1}-C_{3}\right) \cosh 3 \alpha+\left(D_{1}-D_{3}\right) \sinh 3 \alpha\right\} \sin \beta$ $+\left\{-\left(A_{0}-A_{2}\right)+\left(A_{2}-A_{4}\right) \cosh 4 \alpha+\left(B_{2}-B_{4}\right) \sinh 4 \alpha\right\} \cdot \cos 2 \beta$ $+\left\{C_{2}+\left(C_{2}-C_{4}\right) \cosh 4 \alpha+\left(D_{2}-D_{4}\right) \sinh 4 \alpha\right\} \sin 2 \beta$ $+\sum_{n=3}^{n=\infty}\left[-\left(A_{n-2}-A_{n}\right) \cosh (n-2) \alpha-\left(B_{n-2}-B_{n}\right) \sinh (n-2) \alpha\right.$ $\left.+\left(A_{n}-A_{n+2}\right) \cosh (n+2) \alpha+\left(B_{n}-B_{n+2}\right) \sinh (n \mid+2) \alpha\right] \cos n \beta$ $+\sum_{n=3}^{n=\infty}\left[-\left(C_{n-2}-C_{n}\right) \cosh (n-2) \alpha-\left(D_{n-2}-D_{n}\right) \sinh (n-2) \alpha\right.$ $\left.+\left(C_{n}-C_{n+2}\right) \cosh (n+2) \alpha+\left(D_{n}-D_{n+2}\right) \sinh (n+2) \alpha\right] \sin n \beta$. 
We try to satisfy this equation by assuming the following form for $\chi$ :

$$
\chi=\frac{A}{4}[\alpha(\cosh 2 \alpha+\cos 2 \beta)-\sinh 2 \alpha]+\frac{B}{4}[\beta(\cosh 2 \alpha+\cos 2 \beta)-\sin 2 \beta]
$$

$$
+\phi_{0}+\sum_{n=1}^{n=\infty} \phi_{n} \cos n \beta+\sum_{n=1}^{n-\infty} \psi_{n} \sin n \beta+\chi^{\prime}
$$

where $\phi$ 's and $\psi$ 's are functions of $\alpha$ only and $\chi^{\prime}$ is a plane harmonic such as

$$
\begin{aligned}
A^{\prime} \alpha+B^{\prime} \beta & +\sum_{n=1}^{n=\infty}\left(A_{n}^{\prime} \cosh n \alpha+B_{n}^{\prime} \sinh n \alpha\right) \cos n \beta \\
& +\sum_{n=1}^{n=\infty}\left(C_{n}^{\prime} \cosh n \alpha+D_{n}^{\prime} \sinh n \alpha\right) \sin n \beta
\end{aligned}
$$

Substituting in equation (13) and comparing coefficients we get the values of $\phi$ 's and $\psi$ 's:

$$
\begin{aligned}
\phi_{0}= & \frac{1}{4}\left(A_{0}-A_{2}\right) \cosh 2 \alpha-\frac{1}{4} B_{2} \sinh 2 \alpha \\
\phi_{1}= & \frac{1}{8}\left[\left(A_{1}-A_{3}\right) \cosh 3 \alpha+\left(B_{1}-B_{3}\right) \sinh 3 \alpha\right]-B_{1} \alpha \cosh \alpha \\
\phi_{2}= & \frac{1}{4}\left(A_{0}-A_{2}\right)+\frac{1}{12}\left[\left(A_{2}-A_{4}\right) \cosh 4 \alpha+\left(B_{2}-B_{4}\right) \sinh 4 \alpha\right] \\
\phi_{n}= & \frac{1}{4(n-1)}\left[\left(A_{n-2}-A_{n}\right) \cosh (n-2) \alpha+\left(B_{n-2}-B_{n}\right) \sinh (n-2) \alpha\right] \\
& +\frac{1}{4(n+1)}\left[\left(A_{n}-A_{n+2}\right) \cosh (n+2) \alpha+\left(B_{n}-B_{n+2}\right) \sinh (n+2) \alpha\right] \\
\psi_{1}= & \frac{1}{8}\left[\left(C_{1}-C_{3}\right) \cosh 3 \alpha+\left(D_{1}-D_{3}\right) \sinh 3 \alpha\right]+C_{1} \alpha \sinh \alpha, \\
\psi_{2}= & -\frac{1}{4} C_{2}+\frac{1}{12}\left[\left(C_{2}-C_{4}\right) \cosh 4 \alpha+\left(D_{2}-D_{4}\right) \sinh 4 \alpha\right] \\
\psi_{n}= & \frac{1}{4(n-1)}\left[\left(C_{n-2}-C_{n}\right) \cosh (n-2) \alpha+\left(D_{n-2}-D_{n}\right) \sinh (n-2) \alpha\right] \\
& +\frac{1}{4(n+1)}\left[\left(C_{n}-C_{n+2}\right) \cosh (n+2) \alpha+\left(D_{n}-D_{n+2}\right) \sinh (n+2) \alpha\right]
\end{aligned}
$$


It is evident that the terms $\cosh \alpha \cos \beta$ and $\sinh \alpha \sin \beta$ in $\chi^{\prime}$ represent a displacement that is possible in a rigid body.

Hence omitting the rigid body displacement terms and also the terms which ultimately make the stresses many-valued, we can write

where

$$
\begin{aligned}
\chi=A \alpha(\cosh 2 \alpha & +\cos 2 \beta)+B \beta+\phi_{0} \\
& +\sum_{n=1}^{n=\infty} \phi_{n} \cos n \beta+\sum_{n=1}^{n=\infty} \psi_{n} \sin n \beta,
\end{aligned}
$$

$$
\begin{aligned}
\phi_{0} & =a_{0} \cosh 2 \alpha+b \sinh 2 \alpha+c \alpha, \\
\phi_{1} & =a^{\prime} \alpha \cosh \alpha+a_{1} \cosh 3 \alpha+b_{1} \sinh 3 \alpha+b_{1}^{\prime} \sinh \alpha, \\
(16) \quad \phi_{2} & =a_{0}+a_{2} \cosh 4 \alpha+b_{2} \sinh 4 \alpha+a_{2}^{\prime} \cosh 2 \alpha+b_{2}^{\prime} \sinh 2 \alpha, \\
\phi_{n} & =a_{n-2} \cosh (n-2) \alpha+b_{n-2} \sinh (n-2) \alpha+a_{n} \cosh (n+2) \alpha \\
& +b_{n} \sinh (n+2) \alpha+a_{n}^{\prime} \cosh n \alpha+b_{n}^{\prime} \sinh n \alpha,
\end{aligned}
$$

and

$$
\psi_{1}=c^{\prime} \alpha \sinh \alpha+c_{1} \cosh 3 \alpha+d_{1} \sinh 3 \alpha+c_{1}^{\prime} \cosh \alpha
$$

$$
\psi_{2}=c_{0}+c_{2} \cosh 4 \alpha+d_{2} \sinh 4 \alpha+c_{2}^{\prime} \cosh 2 \alpha+d_{2}^{\prime} \sinh 2 \alpha,
$$$$
\psi_{n}=c_{n-2} \cosh (n-2) \alpha+d_{n-2} \sinh (n-2) \alpha+c_{n} \cosh (n+2) \alpha
$$

$$
+d_{n} \sinh (n+2) \alpha+c_{n}^{\prime} \cosh n \alpha+d_{n}^{\prime} \sinh n \alpha \text {. }
$$

4. Even when $\chi$ is known the displacements $u$ and $v$ can only be obtained by first determining $P$ which satisfies equations (10) and (11). Now, instead of finding $P$ directly, it will be convenient to calculate

$$
\frac{1}{h^{2}} \frac{\partial P}{\partial \alpha} \text { and } \frac{1}{h^{2}} \frac{\partial P}{\partial \beta}
$$

subject to the conditions (10) and (11).

It will subsequently be shown that the terms

$$
\alpha(\cosh 2 \alpha+\cos 2 \beta), \alpha \cosh \alpha \cos \beta \text { and } \alpha \sinh \alpha \sin \beta
$$

give rise to many-valued displacements, so that omitting them from $\chi$ and taking into account the fact that $P$ is a harmonic function we can write 
and

$$
\begin{aligned}
\frac{1}{h^{2}} \frac{\partial P}{\partial \alpha}= & A_{0} \sinh 2 \alpha+D_{0} \cosh 2 \alpha+\left(B^{\prime} \cosh \alpha+A_{1} \sinh 3 \alpha\right. \\
& \left.+B_{1} \cosh 3 \alpha\right) \sin \beta+\left(C \sinh \alpha+C_{1} \sinh 3 \alpha+D_{1} \cosh 3 \alpha\right) \cos \beta \\
& +\left(B_{0}+A_{2} \sinh 4 \alpha+B_{2} \cosh 4 \alpha\right) \sin 2 \beta+\left(-D_{0}+C_{2} \sinh 4 \alpha\right. \\
& \left.+D_{2} \cosh 4 \alpha\right) \cos 2 \beta+\sum_{n=3}^{n=\infty}\left[\left\{-A_{n-2} \sinh (n-2) \alpha\right.\right. \\
& \left.-B_{n-2} \cosh (n-2) \alpha+A_{n} \sinh (n+2) \alpha+B_{n} \cosh (n+2) \alpha\right\} \sin n \beta \\
& +\left\{-C_{n-2} \sinh (n-2) \alpha-D_{n-2} \cosh (n-2) \alpha+C_{n} \sinh (n+2) \alpha\right. \\
& \left.\left.+D_{n} \cosh (n+2) \alpha\right\} \cos n \beta\right],
\end{aligned}
$$

$$
\begin{aligned}
\frac{1}{h^{2}} \frac{\partial P}{\partial \beta}= & -B_{0} \sinh 2 \alpha+C_{0} \cosh 2 \alpha+\left(-B^{\prime} \sinh \alpha+A_{1} \cosh 3 \alpha\right. \\
& \left.+B_{1} \sinh 3 \alpha\right) \cos \beta+\left(C \cosh \alpha-C_{1} \cosh 3 \alpha-D_{1} \sinh 3 \alpha\right) \sin \beta \\
& +\left(-C_{0}+A_{2} \cosh 4 \alpha+B_{2} \sinh 4 \alpha\right) \cos 2 \beta+\left(A_{0}-C_{2} \cosh 4 \alpha\right. \\
& \left.-D_{2} \sinh 4 \alpha\right) \sin 2 \beta+\sum_{n=3}^{n=\infty}\left[\left\{-A_{n-2} \cosh (n-2) \alpha\right.\right. \\
& -B_{n-2} \sinh (n-2) \alpha+A_{n} \cosh (n+2) \alpha \\
& \left.+B_{n} \sinh (n+2) \alpha\right\} \cos n \beta+\left\{C_{n-2} \cosh (n-2) \alpha\right. \\
& \left.\left.+D_{n-2} \sinh (n-2) \alpha-C_{n} \cosh (n+2) \alpha-D_{n} \sinh (n+2) \alpha\right\} \sin n \beta\right] .
\end{aligned}
$$

Here the general plane harmonic function $P$ has been differentiated with respect to $\alpha$ and $\beta$ and multiplied by $1 / h^{2}$.

Now substituting in (11) and equating coefficients as usual, we find the values of the coefficients $A_{n}, B_{n}, C_{n}, D_{n}, \cdots$, as follows:

where

$$
\begin{aligned}
& B_{0}=-2 k a_{0}, \quad C_{0}=2 k b, \quad D_{0}=-2 k c_{0}, \\
& A_{n}=2 k b_{n}, \quad B_{n}=2 k a_{n}, \quad C_{n}=-2 k d_{n}, \quad D_{n}=-2 k c_{n},
\end{aligned}
$$

$$
k=\frac{\lambda+2 \mu}{\lambda+\mu} .
$$

In this way all the constants $A_{n}, B_{n}, C_{n}, D_{n}$ can be determined except $A_{0}, B^{\prime}$ and $C$. The terms containing $A_{0}, B^{\prime}$ and $C$ give rise to a displacement which has the following components parallel to axes of $x$ and $y$ respectively:

$$
\frac{1}{2 \mu c^{2}}\left(-B^{\prime} c-2 A_{0} y\right) \text { and } \frac{1}{2 \mu c_{2}}\left(C c+2 A_{0} x\right) \text {, }
$$


thus showing that the displacement is one possible in a rigid body. So we can omit $A_{0}, B^{\prime}$ and $C$. There still remain terms in $\chi$, e.g. those involving $a^{\prime}, c^{\prime}$ and $A$ which are not represented in the differential coefficients $\left(1 / h^{2}\right)(\partial P / \partial \alpha)$ and $\left(1 / h^{2}\right)(\partial P / \partial \beta)$. It is necessary to consider them separately. For the term $\alpha \cosh \alpha \cos \beta$ in $\chi$, let us assume

$$
\begin{aligned}
& \frac{1}{h^{2}} \frac{\partial P}{\partial \alpha}=A^{\prime}(\beta \sinh \alpha \cos \beta-\alpha \cosh \alpha \sin \beta), \\
& \frac{1}{h^{2}} \frac{\partial P}{\partial \beta}=A^{\prime}(\beta \cosh \alpha \sin \beta+\alpha \sinh \alpha \cos \beta) .
\end{aligned}
$$

It can easily be shown that these satisfy

and that

$$
\nabla_{1}^{2} P=0
$$

$$
\frac{\partial}{\partial \alpha}\left(\frac{1}{h^{2}} \frac{\partial P}{\partial \beta}\right)+\frac{\partial}{\partial \beta}\left(\frac{1}{h^{2}} \frac{\partial P}{\partial \alpha}\right)=2 A^{\prime} \sinh \alpha \cos \beta .
$$

$A^{\prime}$ can thus be determined.

Similarly for the other term $\alpha \sinh \alpha \sin \beta$, we can take

$$
\begin{aligned}
& \frac{1}{h^{2}} \frac{\partial P}{\partial \alpha}=B^{\prime}(\alpha \sinh \alpha \cos \beta+\beta \cosh \alpha \sin \beta), \\
& \frac{1}{h^{2}} \frac{\partial P}{\partial \beta}=B^{\prime}(\alpha \cosh \alpha \sin \beta-\beta \sinh \alpha \cos \beta) .
\end{aligned}
$$

For the term $\alpha(\cosh 2 \alpha+\cos 2 \beta)$, we take

$$
\begin{aligned}
& \frac{1}{h^{2}} \frac{\partial P}{\partial \alpha}=c^{\prime}(\beta \sinh 2 \alpha-\alpha \sin 2 \beta), \\
& \frac{1}{h^{2}} \frac{\partial P}{\partial \beta}=c^{\prime}(\beta \sin 2 \beta+\alpha \sinh 2 \alpha) .
\end{aligned}
$$

They satisfy equation (10) and give

$$
\frac{\partial}{\partial \alpha}\left(\frac{1}{h^{2}} \frac{\partial P}{\partial \beta}\right)+\frac{\partial}{\partial \beta}\left(\frac{1}{h^{2}} \frac{\partial P}{\partial \alpha}\right)=2 c^{\prime} \sinh 2 \alpha+2 c^{\prime} \alpha(\cosh 2 \alpha-\cos 2 \beta) .
$$

From (11) it is easily seen that

$$
c^{\prime}=\frac{2(\lambda+2 \mu)}{\lambda+\mu} A .
$$


The stresses can be easily calculated from (8) as below:

$\frac{2}{c^{2}} \frac{\widehat{\alpha \alpha}}{h^{4}}=\left(3 \phi_{2}+\phi_{0}^{\prime} \sinh 2 \alpha\right)+\left(-\phi_{1} \cosh 2 \alpha+\phi_{1}^{\prime} \sinh 2 \alpha+6 \phi_{3}\right) \cos \beta$

$+\left(-\psi_{1} \cosh 2 \alpha+\psi_{1}^{\prime} \sinh 2 \alpha+6 \psi_{3}\right) \sin \beta-B \sin 2 \beta$

$+\sum_{n=2}^{n=\infty}\left[\left\{-n^{2} \phi \cosh 2 \alpha+\phi_{n}^{\prime} \sinh 2 \alpha+\frac{1}{2}(n+2)(n+3) \phi_{n+2}\right.\right.$

$\left.+\frac{1}{2}(n-2)(n-3) \phi_{n-2}\right\} \cos n \beta+\left\{n^{2} \psi_{n} \cosh 2 \alpha+\psi_{n}^{\prime} \sinh 2 \alpha\right.$

$\left.\left.+\frac{1}{2}(n+2)(n+3) \psi_{n+2}+\frac{1}{2}(n-2)(n-3) \psi_{n-2}\right\} \sin n \beta\right]$;

$\frac{2}{c^{2}} \frac{\widehat{\beta \beta}}{h^{4}}=\phi_{0}{ }^{\prime \prime} \cosh 2 \alpha-\phi_{0}^{\prime} \sinh 2 \alpha-\frac{1}{2} \phi_{2}{ }^{\prime}-\phi_{2}$

$+\left(\phi_{1}^{\prime \prime} \cosh 2 \alpha-\phi_{1}^{\prime} \sinh 2 \alpha-\frac{1}{2} \phi_{3}{ }^{\prime \prime}-\frac{3}{2} \phi_{3}\right) \cos \beta$

$+\left(\psi_{1}^{\prime \prime} \cosh 2 \alpha-\psi_{1}^{\prime} \sinh 2 \alpha-\frac{1}{2} \psi_{3}{ }^{\prime \prime}-\frac{3}{2} \psi_{3}\right) \sin \beta+B \sin 2 \beta$

(24)

$+\sum_{n=2}^{n=\infty}\left[\left\{-\frac{1}{2} \phi_{n-2}^{\prime \prime}+\frac{1}{2}(n-2) \phi_{n-2}+\phi_{n}^{\prime \prime} \cosh 2 \alpha-\phi_{n}^{\prime} \sinh 2 \alpha\right.\right.$

$\left.-\frac{1}{2} \phi_{n+2}^{\prime \prime}-\frac{1}{2}(n+2) \phi_{n+2}\right\} \cos n \beta+\left\{-\frac{1}{2} \psi_{n-2}^{\prime \prime}+\frac{1}{2}(n-2) \psi_{n-2}\right.$

$\left.\left.+\psi_{n}^{\prime \prime} \cosh 2 \alpha-\psi_{n}^{\prime} \sinh 2 \alpha-\frac{1}{2} \psi_{n+2}^{\prime \prime}-\frac{1}{2}(n+2) \psi_{n+2}\right\} \sin n \beta\right]$;

and

$\frac{2}{c^{2}} \frac{\widehat{\alpha \beta}}{h^{4}}=B \sinh 2 \alpha+\left(\phi_{1}^{\prime} \cosh 2 \alpha-\phi_{1} \sinh 2 \alpha-2 \phi_{3}^{\prime}\right) \sin \beta+\left(-\psi_{1}^{\prime} \cosh 2 \alpha\right.$ $\left.+\psi_{1} \sinh 2 \alpha+2 \psi_{3}^{\prime}\right) \cos \beta+\left(\phi_{0}^{\prime}+2 \phi_{2}^{\prime} \cosh 2 \alpha-2 \phi_{2} \sinh 2 \alpha\right.$ $\left.-\frac{5}{2} \phi_{4}^{\prime}\right) \sin 2 \beta+\left(-2 \psi_{2}^{\prime} \cosh 2 \alpha+2 \psi_{2} \sinh 2 \alpha+\frac{5}{2} \psi_{4}^{\prime}\right) \cos 2 \beta$

$+\sum_{n=3}^{n=\infty}\left[\left\{-\frac{1}{2}(n-3) \phi_{n-2}^{\prime}+n \phi_{n}^{\prime} \cosh 2 \alpha-n \phi_{n} \sinh 2 \alpha\right.\right.$

$\left.-\frac{1}{2}(n+3) \phi_{n+2}^{\prime}\right\} \sin n \beta+\left\{\frac{1}{2}(n-3) \psi_{n-2}^{\prime}-n \psi_{n}^{\prime} \cosh 2 \alpha\right.$

$\left.\left.+n \psi_{n} \sinh 2 \alpha+\frac{1}{2}(n+3) \psi_{n+2}^{\prime}\right\} \cos n \beta\right]$. 
Here we have omitted the first term in $\chi$ given in (15) while calculating the stresses.

5. We shall now consider some applications of the above solutions.

(a). Let an infinite solid with an elliptic cylindrical hollow be subjected to a pressure $P_{1}$ on the inner elliptic boundary.*

Assume in this case

$$
\chi=A\left(e^{-2 \alpha}+\cos 2 \beta\right)+B \alpha .
$$

Then we have

and

$$
\begin{aligned}
\frac{c^{2}}{2}(\cosh 2 \alpha-\cos 2 \beta)^{2} \widetilde{\alpha \alpha}= & \left\{A\left(3-2 e^{-2 \alpha} \sinh 2 \alpha\right)+B \sinh 2 \alpha\right\} \\
& -4 A \cosh 2 \alpha \cos 2 \beta+A \cos 4 \beta,
\end{aligned}
$$

$$
\frac{c^{2}}{2}(\cosh 2 \alpha-\cos 2 \beta)^{2} \widehat{\alpha \beta}=(B-2 A \cosh 2 \alpha) \sin 2 \beta .
$$

The conditions on the boundary give

also

$$
\widehat{\alpha \alpha}=-P_{1} \text { and } \overparen{\alpha \beta}=0 \text { when } \alpha=\alpha_{1},
$$

$$
\overparen{\alpha \alpha}=0 \quad \text { and } \overparen{\alpha \beta}=0 \text { when } \alpha=\infty
$$

These give

$$
A=-\frac{P_{1} c^{2}}{4} \text { and } B=-\frac{P_{1} c^{2}}{2} \cosh 2 \alpha_{1}
$$

In this case $P$ can be easily shown to be equal to

$$
-\frac{\lambda+2 \mu}{\lambda+\mu} P_{1} \tan ^{-1}\left(\frac{e^{-2 \alpha} \sin 2 \beta}{1-e^{-2 \alpha} \cos 2 \beta}\right) .
$$

Equations (9) now give the following displacements:

$$
\begin{aligned}
& \frac{u}{h}=\frac{P_{1} c^{2}}{4(\lambda+\mu)} e^{-2 \alpha}-\frac{\lambda+2 \mu}{4 \mu(\lambda+\mu)} P_{1} c^{2} \cos 2 \beta+\frac{P_{1} c^{2}}{4 \mu} \cosh 2 \alpha_{1}, \\
& \frac{v}{h}=\frac{P_{1} c^{2}}{4(\lambda+\mu)} \sin 2 \beta .
\end{aligned}
$$

(b). For the second case we are going to consider let us assume

* This case differs from the cases studied by Kolosoff and Pöschl in boundary conditions only. In Pöschl's case $\chi=$ const. and $\partial \chi / \partial \alpha=0$ on the inner boundary $\alpha=\alpha_{1}$ and $\chi \rightarrow(1 / 16) p c^{2} e^{2 \alpha}[1+\cos$ $\left.2\left(\beta-\beta^{\prime}\right)\right]$ as $\alpha \longrightarrow \infty$. 
(29) $\chi=\left(A+A^{\prime} \alpha\right)(\cosh 2 \alpha+\cos 2 \beta)+B \sinh 2 \alpha$

$+c \alpha+(D \cosh 2 \alpha+E \sinh 2 \alpha) \cos 2 \beta$.

If the boundaries $\alpha=\alpha_{1}$ and $\alpha=\alpha_{2}$ be subjected to pressures $P_{1}$ and $P_{2}$ respectively, we easily find by calculating the stresses and taking into account the boundary conditions the values

$$
\begin{aligned}
A= & -\frac{1}{4 M} c^{2}\left[P_{1}\left\{\alpha_{2}-\frac{1}{2} \tanh \left(\alpha_{2}-\alpha_{1}\right)\right\}-P_{2}\left\{\alpha_{1}-\frac{1}{2} \tanh \left(\alpha_{1}-\alpha_{2}\right)\right\}\right] \\
A^{\prime} & =\frac{1}{4 M}\left(P_{1}-P_{2}\right) c^{2} \\
B & =-\frac{1}{4 M}\left(P_{1}-P_{2}\right) c^{2} \\
\text { (30) } \quad C & =\frac{1}{4 M}\left(P_{1}-P_{2}\right) c^{2} \frac{\cosh \left(\alpha_{2}+\alpha_{1}\right)}{\cosh \left(\alpha_{2}-\alpha_{1}\right)} \\
D & =\frac{1}{8 M}\left(P_{1}-P_{2}\right) c^{2} \frac{\sinh \left(\alpha_{2}+\alpha_{1}\right)}{\cosh \left(\alpha_{2}-\alpha_{1}\right)} \\
E & =-\frac{1}{8 M}\left(P_{1}-P_{2}\right) c^{2} \frac{\cosh \left(\alpha_{2}+\alpha_{1}\right)}{\cosh \left(\alpha_{2}-\alpha_{1}\right)}
\end{aligned}
$$

where

$$
M=\left(\alpha_{2}-\alpha_{1}\right)-\tanh \left(\alpha_{2}-\alpha_{1}\right) .
$$

Moreover, we have in this case

$$
\begin{aligned}
\frac{1}{h^{2}} \frac{\partial P}{\partial \alpha}= & \frac{2(\lambda+2 \mu)}{\lambda+\mu}\left[A^{\prime}(\beta \sinh 2 \alpha-\alpha \sin 2 \beta)-A \sin 2 \beta \mid\right. \\
\frac{1}{h^{2}} \frac{\partial P}{\partial \beta}= & \frac{2(\lambda+2 \mu)}{\lambda+\mu}\left[A^{\prime}(\beta \sin 2 \beta+\alpha \sinh 2 \alpha)+A \sinh 2 \alpha\right. \\
& +B(\cosh 2 \alpha-\cos 2 \beta)] .
\end{aligned}
$$

Also the displacements are given by

$$
\begin{aligned}
\frac{u}{h} & =\frac{1}{\lambda+\mu}\left[A \sinh 2 \alpha+\left(B-\frac{\lambda+\mu}{\mu} A^{\prime}\right) \cosh 2 \alpha+\frac{\lambda+2 \mu}{\mu} A^{\prime} \beta \sin 2 \beta\right. \\
& \left.+A^{\prime} \alpha \sinh 2 \alpha-\left(\frac{\lambda+2 \mu}{\mu} B+\frac{\lambda+\mu}{2 \mu} A^{\prime}\right) \cos 2 \beta\right]-\frac{C}{2 \mu} \\
& -\frac{1}{\mu}(D \sinh 2 \alpha+E \cosh 2 \alpha) \cos 2 \beta
\end{aligned}
$$

and 


$$
\begin{aligned}
\frac{v}{h}= & \frac{1}{\lambda+\mu}\left[\frac{\lambda+2 \mu}{\mu} A^{\prime} \beta \sinh 2 \alpha-A^{\prime} \alpha \sin 2 \beta-A \sin 2 \beta\right] \\
& +\frac{1}{\mu}(D \cosh 2 \alpha+E \sinh 2 \alpha) \sin 2 \beta
\end{aligned}
$$

These displacements are not single-valued. Considering only the manyvalued terms, we write

$$
\begin{aligned}
& \frac{u^{\prime}}{h}=\frac{\lambda+2 \mu}{\mu(\lambda+\mu)} A^{\prime} \beta \sin 2 \beta, \\
& \frac{v^{\prime}}{h}=\frac{\lambda+2 \mu}{\mu(\lambda+\mu)} A^{\prime} \beta \sinh 2 \alpha .
\end{aligned}
$$

If we restrict $\beta$ by a convention such as $0<\beta<2 \pi$, these displacements become single-valued and discontinuous. When $\beta=0$, we have $u^{\prime}=0$ and $v^{\prime}=0$; when $\beta=2 \pi$, we have

$$
u^{\prime}=0 \quad \text { and } \quad v^{\prime}=\frac{4 \pi(\lambda+2 \mu)}{\mu(\lambda+\mu)} \frac{A^{\prime}}{c} \cosh \alpha .
$$

Therefore there is no discontinuity in the value of $u$, but the value of $v$ decreases suddenly by the amount $v_{0}$ given by

$$
v_{0}=\frac{4 \pi(\lambda+2 \mu)}{\mu(\lambda+\mu)} \frac{A^{\prime}}{c} \cosh \alpha
$$

as $\beta$ increases from $2 \pi-0$ to $2 \pi+0$. This is the jump in the value of $v$ on the positive $x$-axis.

Now when $\beta=0$,

Therefore

$$
x=x_{0}=c \cosh \alpha .
$$

$$
\frac{v_{0}}{x_{0}}=\frac{4 \pi(\lambda+2 \mu)}{\mu(\lambda+\mu)} \frac{A^{\prime}}{c^{2}}=\text { constant } .
$$

This result we can interpret as a dislocation* due to an axial wedgeshaped fissure bounded by the planes

$$
y= \pm \frac{2 \pi(\lambda+2 \mu)}{\mu(\lambda+\mu)} \frac{A^{\prime}}{c^{2}} x
$$

where $A^{\prime}$ is given by (30).

* For the theory of dislocation, see Volterra's paper Sur l'élquilibre des corps élastiques multiplement connexes, Annales de l'Ecole Normale Supérieure, (3), vol. 24 (1907), pp. 401-517. See also Love's Theory of Elasticity (3d edition), p. 219. 
Hence the assumption made in (29) solves the problem of dislocation for a wedge-shaped fissure in a body bounded by two confocal elliptic cylinders.

(c). As a third application let us consider the problem dealt with by Love.*

Let an infinite solid be bounded internally by an elliptic boundary of semiaxes $a$ and $b$ and let the inner boundary be turned through an angle $\phi$.

Let $\alpha=\alpha_{1}$ be the inner boundary. Then the displacement at any point on the boundary is $r \phi$, perpendicular to the radius vector drawn from the center of the elliptic section to the point, where $r=$ distance of the point from the center. This displacement has a component $-c \phi \sinh \alpha \sin \beta$ parallel to $O x$ and $c \phi \cosh \alpha \cos \beta$ parallel to $O y$. If $u, v$ be the displacements along and perpendicular to the normal to $\alpha=$ constant we have when $\alpha=\alpha_{1}$

Assume

$$
\begin{aligned}
& \frac{u}{h}=\frac{1}{2} c^{2} \phi \sin 2 \beta, \\
& \frac{v}{h}=\frac{1}{2} c^{2} \phi \sinh 2 \alpha_{1} .
\end{aligned}
$$

$$
\chi=A \beta+B \sin 2 \beta+C e^{-2 \alpha} \sin 2 \beta
$$

Then

$$
\begin{aligned}
& \frac{1}{h^{2}} \frac{\partial P}{\partial \alpha}=A_{0} \sinh 2 \alpha-\frac{2(\lambda+2 \mu)}{\lambda+\mu} B(\cosh 2 \alpha-\cos 2 \beta), \\
& \frac{1}{h^{2}} \frac{\partial P}{\partial \beta}=A_{0} \sin 2 \beta
\end{aligned}
$$

and

$$
\begin{aligned}
2 \mu \frac{u}{h}= & 2 C e^{-2 \alpha} \sin 2 \beta+A_{0} \sin 2 \beta \\
2 \mu \frac{v}{h}= & -A+A_{0} \sinh 2 \alpha-\frac{2(\lambda+2 \mu)}{\lambda+\mu} B \cosh 2 \alpha \\
& +\frac{2 \mu}{\lambda+\mu} B \cos 2 \beta-2 C e^{-2 \alpha} \cos 2 \beta .
\end{aligned}
$$

From the boundary conditions (37) and from the condition that $u$ and $v$ vanish at infinity we find

\footnotetext{
*Theory of Elasticity (3d edition), p. 275.
} 
(41)

$$
\begin{aligned}
A & =-\frac{\mu(\lambda+2 \mu)}{\lambda+3 \mu} c^{2} \phi e^{-2 \alpha_{1}}-\mu c^{2} \phi \sinh 2 \alpha_{1}, \\
B & =\frac{1}{2} \frac{\mu(\lambda+\mu)}{\lambda+3 \mu} c^{2} \phi \\
C & =\frac{1}{2} \frac{\mu^{2}}{\lambda+3 \mu} c^{2} \phi e^{2 \alpha_{1}}, \\
A_{0} & =\frac{\mu(\lambda+2 \mu)}{\lambda+3 \mu} c^{2} \phi .
\end{aligned}
$$

Then

$$
\frac{u}{h}=\frac{1}{2} \frac{\mu}{\lambda+3 \mu} c^{2} \phi e^{2 \alpha_{1}}\left[e^{-2 \alpha}+\frac{\lambda+2 \mu}{\mu} e^{-2 \alpha_{1}}\right] \sin 2 \beta,
$$

$$
\frac{v}{h}=\frac{1}{2} c^{2} \phi \sinh 2 \alpha_{1}+\frac{1}{2} c^{2} \phi e^{2 \alpha_{1}}\left(e^{-2 \alpha_{1}}-e^{-2 \alpha}\right)\left\{\frac{\lambda+2 \mu}{\lambda+3 \mu} e^{-2 \alpha_{1}}+\frac{\mu}{\lambda+3 \mu} \cos 2 \beta\right\} .
$$

But $a=c \cosh \alpha_{1}$ and $b=c \sinh \alpha_{1}$, and consequently

$$
\begin{aligned}
\frac{u}{h}= & \frac{1}{2} \frac{\mu}{\lambda+3 \mu}(a+b)^{2} \phi\left\{e^{-2 \alpha}+\frac{\lambda+2 \mu}{\mu} \frac{a-b}{a+b}\right\} \sin 2 \beta, \\
\frac{v}{h}= & a b \phi+\frac{1}{2}(a+b)^{2} \phi\left(\frac{a-b}{a+b}-e^{-2 \alpha}\right)\left\{\frac{\lambda+2 \mu}{\lambda+3 \mu} \frac{a-b}{a+b}\right. \\
& \left.+\frac{\mu}{\lambda+3 \mu} \cos 2 \beta\right\} .
\end{aligned}
$$

This is the same result as found by Love.

In conclusion I wish to express my thanks to Dr. N. R. Sen for guidance and help in course of the work.

University College of Science,

$$
\text { Calcutta, India }
$$

